\title{
Gold nanostars for superficial diseases: a promising tool for localized hyperthermia?
}

\section{"...medical hyperthermia has been revisited recently thanks to the ability to exploit nanotechnology to obtain in situ treatments and high heat release efficiency."}

\section{KEYWORDS: gold nanoparticle - hyperthermia $\approx$ near-infrared radiation " plasmon resonance}

Superficial diseases, such as mycoses, fungal infections and skin cancers, primarily affect the skin. The latter includes melanomas or basal cell carcinoma and squamous cell carcinoma [1]. Superficial recurrences of breast cancer, particularly in patients that have previously been irradiated [2] are also common. An impressive number of skin diseases, even when they are not fatal, severely affect patients from a physiological and psychological point of view. Therefore, there is a clear need for rapid and noninvasive methods to diagnose and treat these diseases. Ideally, one would be highly selective in addressing lesions by means of localized treatment protocols, such as localized drug release and hyperthermia. Indeed, medical hyperthermia has been revisited recently thanks to the ability to exploit nanotechnology to obtain in situ treatments and high heat release efficiency $[3,4]$. Wholebody hyperthermia has, in fact, been used for a long time in cancer therapy [5]. Limiting our analysis to skin treatments, a sensible reduction in the apoptosis of keratinocytes induced by UVB has been observed in murine skin under mild local hyperthermia [6]. In normal human skin in vivo, hyperthermia upregulates heatshock proteins and thereby induces a physiological response that protects skin from UV light [7]. Hyperthermia has also been reported to induce apoptosis in two types of skin cancer cells through endoplasmic reticulum-mediated apoptosis [1].

Combined hyperthermic/chemotherapeutic or radiotherapeutic methods are particularly effective. Chemohyperthermic treatments of carcinomas and sarcomas have been carried out with whole-body protocols and using isolated regional therapy $[8,9]$. Superficial breast metastases and Hodgkin's lymphomas have been treated with a combination of hyperthermia and radiation therapy [2].
Despite the encouraging results of these pioneering studies, whole-body treatments in the fever temperature range are inefficient and isolated regional therapies are complex [9]. They would greatly benefit from the use of novel approaches, such as those based on nanostructured materials. For years, nanotechnology has been suggested to improve topical treatments [10]. In fact, the skin plays a double role in the novel field of nanomedicine: nanocompounds can enhance efficiency and limit drawbacks of topical treatments, acting as adjuvants of topical therapies, and skin diseases can be treated locally by nanostructured materials, such as nanoparticles (NPs). Although the use of lipid-based NPs has been favored owing to their affinity to the skin composition [11], metalbased NPs, decorated with stealth polymers and antigen-recognizing molecules, are now being considered for application. Metal NPs can interact with skin at a cellular level to enhance immune reactivity for vaccine applications [12]. Treatment of skin with silver NPs has been used against microbial infections [13], since the NPs provide a slow release of antimicrobial silver ions that have a positive role in chronic wound healing. In addition, noble metal NPs can behave as localized thermal loaders [14] and, therefore, would allow physical treatments (thermalinduced apoptosis) to be coupled to the chemical therapy. Gold nonspherical NPs are particularly interesting in this sense due to their capability to locally release heat efficiently when irradiated in the near-infrared (NIR) region [3], a window (650-900 $\mathrm{nm}$ ) of the electromagnetic spectrum where skin is particularly transparent. An increasing number of studies suggest that combined chemical (drug-delivering NPs) and physical (NP-assisted hyperthermia) treatments based on metal NPs can be beneficial for infectious diseases and cancers.

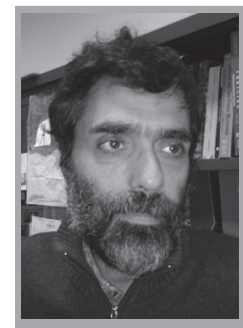

Giuseppe Chirico

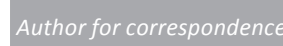
Dipartimento di Fisica, Università degli Studi di Milano-Bicocca, Milano, Italy giberto.chirico@mib.infn.it

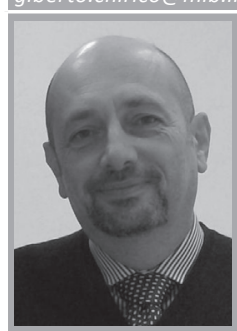

Piersandro Pallavicini Dipartimento di Chimica, Università degli Studi di Pavia, Pavia, Italy

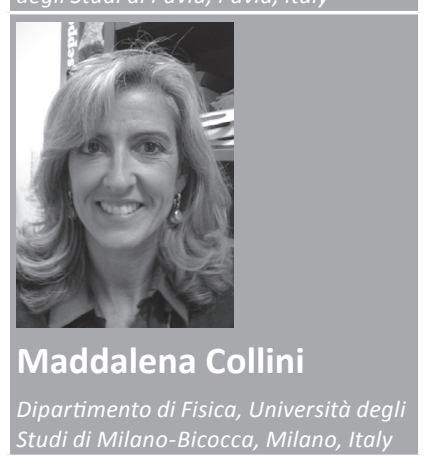


Efforts have concentrated on the tailored synthesis of nonspherical gold NPs [4], as they are endowed with large plasmon resonances in the NIR region of the spectrum, where the wavelength of maximum absorption can be finely tuned. The light absorption in the NIR region of the spectrum corresponds to highly localized (less than $15 \mathrm{~nm} \mathrm{[14])} \mathrm{thermal} \mathrm{release.} \mathrm{Histori-}$ cally, gold nanorods have been the first type of anisotropic gold NPs to be developed and directly applied to hyperthermic treatements in vitro [3] and in vivo [15]. More exotic shapes have now been developed, such as nanocages, nanoprisms and nanocrescents [4]. The interaction of these NPs with the cells $[3,16]$, their toxicity and their fate within the human body [17] are largely affected by the chemical properties (primarily polarity and charge) of their surfaces. In addition, size and shape markedly affect the in vivo behavior of the anisotropic NPs. Their shape anisotropy (axial ratio) essentially determines the position and the amplitude of the NIR plasmon resonance $[4,18]$. However, shape anisotropy is also correlated to an increased overall encumbrance with respect to spherical NPs.

Recently developed gold nanostars (GNSs) [19] can be tuned in shape from sea urchin-like [20] to planar, highly regular, penta-branched stars [18]. This class of NPs is attractive because of their optical and catalytic features, comparable to those of gold nanorods of high axial ratio, and their overall encumbrance is limited $(\sim 20 \mathrm{~nm}$ hydrodynamic radius) $[18,20]$. Similar to other gold NPs, they display plasmonic resonances redshifted with respect to those of gold nanospheres [4]. An intense band in the NIR range (750-1000 $\mathrm{nm}$ ) appears beside the weaker band at $500-550 \mathrm{~nm}$. The NIR plasmon resonance of GNSs can be tuned in a wide NIR range up to $1250 \mathrm{~nm}$ by varying the axial ratio of protruding branches [18], and multiple NIR plasmon resonances can also be obtained. The NIR plasmonic resonances are characterized by excellent transduction of their absorbed light into heat $[14,16]$. The estimated specific absorption rate of sea urchin GNSs can reach $200 \mathrm{~kW} / \mathrm{g}$, a value that is almost two orders of magnitude larger than that typically obtained for magnetite NPs [14]. The shape of the GNSs and their interaction with cells is modulated by the different types of surfactants or coating agents used in their synthesis. These agents also determine the NP surface properties and capabilities to be decorated. In the aqueous seed-growth synthetic protocol for GNSs, the use of the zwitterionic surfactant lauryl sulfobetaine yields easily coatable GNSs due to the surfactant's weak interaction with gold. Other weak coating agents, such as the nonionic surfactant Triton X-100 ${ }^{\mathrm{TM}}$ (TX100; Sigma-Aldrich, MO, USA), result in regular penta-branched GNSs. These can also be easily coated with thiols on their whole surface and show the unprecedented feature of three plasmonic resonances [18]. Two out of the three NIR plasmonic resonances depend strongly on the length:width ratio of the branches, and span the entire NIR range entering the short-wave infrared domain. Both NIR plasmonic resonances efficiently release heat under laser excitation. Gold nanostars synthesized with weakly interacting coating agents are characterized with relative ease by their surface decoration, a property that is not shared by most other gold anisotropic NPs. The possibility of easily decorating the surface of this class of metal NPs is at the basis of their feasible application in medicine. The latter requires limiting toxic effects and enhancing target efficiency. Nanorods are commonly prepared with seed growth syntheses using the cetyltrimethylammonium bromide surfactant, which forms a strongly adhering double layer on the gold surface, resulting in a smooth thiolation only on the short ends of the nanorods, while requiring multistep and more complicated procedures to coat the whole surface. With GNSs synthesized using lauryl sulfobetaine [20] or TX100 [18] as stabilizers, surface decoration is only a matter of adding thiols to the GNS solution, obtaining fast and complete coating. This smoothness also allows easy access to multicomponent coating, simply using a two- or three-component thiol mixture in the coating solution.

\section{"This class of nanoparticles is attractive because of their optical and catalytic features, comparable to those of gold nanorods of high axial ratio, and their overall encumbrance is limited..."}

The GNSs could also be decorated with mixed lipid-polymer coatings by exploiting thiol chemistry. This multiple decoration would increase/modify their interaction with skin and enable the NPs' drug delivery capabilities. Polymers capable of trapping and releasing drugs under temperature control obtained by NIR laser irradiation may in fact be employed for this purpose. By tuning the hydrophobic/electrostatic character of the bonding between toxic drugs and decorating polymers, the drugs could be delivered to the pathology location and released in situ under NIR irradiation control. Toxicology 
issues are relevant for in vivo uses and this feature can also be modulated by surface properties. Few studies have been reported about the interaction between skin and nonbiodegradable NPs. Regarding gold, small-size (2-4 nm) gold NPs functionalized with cationic head groups penetrate into the hydrophobic moiety of the lipid bilayers and cause membrane disruption at increased concentrations. Such a behavior is expected for gold nanorods that are anionic. By contrast, the gold NPs functionalized with anionic head groups or that bear slightly negative charges, interact with the lipid bilayer by dehydrating and shielding it [11] and probably enter the cell through endocytosis. The ease in surface decoration of GNS and their neutral or slightly negative surface charge can be a key feature in reducing toxicology issues in their in vivo application.

In summary, the physical and surface chemistry properties of GNSs make them particularly promising for topical application of hyperthermia to treat skin and superficial diseases. In fact, skin absorption in the NIR-short wave infrared domain is limited and one could achieve selective, localized hyperthermia on the skin surface treated with NPs. Moreover, the ease in surface decoration displayed by GNSs would allow tailoring of their surface for charge and lipid content and, therefore, enhance their efficacy for the treatment of superficial diseases.

\section{Financial \& competing interests disclosure}

The authors have no relevant affiliations or financial involvement with any organization or entity with a financial interest in or financial conflict with the subject matter or materials discussed in the manuscript. This includes employment, consultancies, honoraria, stock ownership or options, expert testimony, grants or patents received or pending, or royalties.

No writing assistance was utilized in the production of this manuscript.

\section{References}

1 Shellman YG, Howe WR, Miller LA, Goldstein NB, Pacheco TR. Hyperthermia induces endoplasmic reticulum-mediated apoptosis in melanoma and non-melanoma skin cancer cells. J. Invest. Derm. 128, 949-956 (2008).

2 Zagar TM, Oleson JR, Vujaskovic Z et al. Hyperthermia combined with radiation therapy for superficial breast cancer and chest wall recurrence: a review of the randomised data. Int. J. Hyperthermia 26, 612-617 (2010).

3 Huang X, Jain PK, El-Sayed IH, El-Sayed MA. Determination of the minimum temperature required for selective photothermal destruction of cancer cells with the use of immunotargeted gold nanoparticles. Photochem. Photobiol. 82, 412-417 (2006).

4 Eustis S, El-Sayed MA. Why gold nanoparticles are more precious than pretty gold: noble metal surface plasmon resonance and its enhancement of the radiative and nonradiative properties of nanocrystals of different shapes. Chem. Soc. Rev. 35, 209-217 (2006).

5 Wust P, Hildebrandt B, Sreenivasa G et al. Hyperthermia in combined treatment of cancer. Lancet Oncol. 3, 487-497 (2002).

6 Kane KS and Maytin EV. Ultraviolet $\mathrm{B}$-induced apoptosis of keratinocytes in murine skin is reduced by mild local hyperthermia. J. Invest. Derm. 104, 62-67 (1995).

7 Wilson N, McArdle A, Guerin D, Tasker H. Hyperthermia to normal human skin in vivo upregulates heat shock proteins $27,60,72 \mathrm{i}$ and 90. J. Cutan. Path. 27, 176-182 (2000).

8 Bull JM, Scott GL, Strebel FR et al. Fever-range whole-body thermal therapy combined with cisplatin, gemcitabine, and daily interferonalpha: a description of a Phase I-II protocol. Int. J. Hyperthermia 24, 649-662 (2008).

9 Duprat JP, Domingues AL, Coelho EG et al. Long-term response of isolated limb perfusion with hyperthermia and chemotherapy for Merkel cell carcinoma. Eur. J. Surg. Oncol. 35, 568-572 (2009).

10 Peer D, Karp JM, Hong S, Farokhzad OC, Margalit R, Langer R. Nanocarriers as an emerging platform for cancer therapy. Nat. Nanotechnol. 2, 751-760 (2007).

11 Tatur S, Maccarini M, Barker R, Nelson A, Fragneto G. Effect of functionalized gold nanoparticles on floating lipid bilayer. Langmuir 29, 6606-6614 (2013).

12 Prow TW, Chen X, Prow NA et al. Nanopatch-targeted skin vaccination against West Nile Virus and Chikungunya virus in mice. Small 6, 1776-1784 (2010).

13 Taglietti A, Fernandez YA, Amato et al. Antibacterial activity of glutathione-coated silver nanoparticles against Gram positive and Gram negative bacteria. Langmuir 28 , 8140-8148 (2012).
14 Freddi S, Sironi L, D’Antuono R et al. A molecular thermometer for nanoparticles for optical hyperthermia. Nano Lett. 13, 2004-2010 (2013).

15 Hirsch LR, Stafford RJ, Bankson JA et al. Nanoshell-mediated near-infrared thermal therapy of tumors under magnetic resonance guidance. Proc. Natl Acad. Sci USA 100, 13549-13554 (2003).

16 Sironi L, Freddi S, Caccia M et al. Gold branched nanoparticles for cellular treatments. J. Phys. Chem. C 116, 18407-18418 (2012).

$17 \mathrm{Li} \mathrm{YF}$, Chen C. Fate and toxicity of metallic and metal-containing nanoparticles for biomedical applications. Small 7, 2965-2980 (2011).

18 Pallavicini $\mathrm{P}$, Donà $\mathrm{A}$, Casu $\mathrm{A}$ et al. Triton X-100 for three-plasmon gold nanostars with two photothermally active NIR (near IR) and SWIR (short-wavelength IR) channels. Chem. Commun. 49, 6256-6267 (2013).

19 Guerrero-Martínez A, Barbosa S, Pastoriza-Santos I, Liz-Marzán LM. Nanostars shine bright for you. Colloidal synthesis, properties and applications of branched metallic nanoparticles. Curr. Opin. Colloid Inter. Sci. 16, 118-127 (2011).

20 Pallavicini P, Chirico G, Collini M et al. Synthesis of branched Au nanoparticles with tunable near-infrared LSPR using a zwitterionic surfactant. Chem. Commun. 47, 1315-1317 (2011). 